\title{
SINC-COLLOCATION METHOD WITH ORTHOGONALIZATION FOR SINGULAR POISSON-LIKE PROBLEMS
}

\author{
GUANGYAN YIN
}

\begin{abstract}
This paper uses the Sinc-collocation method to solve singular Poisson-like problems (a first- or higher-order partial derivative of the exact solution is unbounded on the boundary). A linear system is obtained which is the same as that obtained by using the Sinc-Galerkin method. With a smart choice of the stepsize and the number of the gridpoints, the orthogonalization technique is successfully applied to solve the linear system obtained, and a numerical approximation is obtained with an exponential accuracy $O\left(\exp \left(-c N^{\frac{1}{2}}\right)\right)$, where $N$ is a truncation parameter and $c$ is a constant independent of $N$.
\end{abstract}

\section{INTRODUCTION}

We first consider the two-dimensional Poisson problem $(P P)$ :

$$
\left\{\begin{array}{l}
\frac{\partial^{2} u}{\partial x^{2}}+\frac{\partial^{2} u}{\partial y^{2}}=f(x, y), \quad(x, y) \in \Omega, \\
\left.u\right|_{\partial \Omega}=0
\end{array}\right.
$$

where the domain $\Omega \equiv\left(a_{1}, b_{1}\right) \times\left(a_{2}, b_{2}\right)$, and where the first- or higher-order partial derivative of the exact solution is unbounded on the boundary. The standard finite difference or finite element method will experience some difficulties with the above singular $P P$ [12]. To overcome the difficulties, Stenger $[9,10]$ and Lund et al. $[3,4]$ used the Sinc-Galerkin method to solve $P P$ by finding the inverse of the matrix associated with the Sylvester equation, and thus obtained the numerical solutions with an accuracy of exponential order $O\left(\exp \left(-c N^{\frac{1}{2}}\right)\right)$.

In this paper, we use the Sinc-collocation method with the orthogonalization technique to establish a scheme to solve $P P$ and obtain a numerical solution with an accuracy of the same exponential order $O\left(\exp \left(-c N^{\frac{1}{2}}\right)\right)$.

In $\S 2$ we review some basic facts about the Sinc approximation and derive useful Sinc-collocation formulas in two dimensions.

In $\S 3$ we apply the Sinc-collocation method for $P P$ to construct a numerical scheme and obtain the Sylvester equation, which is the same as that obtained by using an entirely different procedure (the Sinc-Galerkin method) [4]. Then we prove that if a smart choice is made of the stepsize and the gridpoint number, the Sylvester equation can be solved by an orthogonalization technique, which permits a significant reduction in both storage and computation compared with

Received by the editor February 4, 1992 and, in revised form, June 22, 1992.

1991 Mathematics Subject Classification. Primary 65N35, 65N99, 65F25. 
what would be required by traditional methods. Some numerical results are given in the same section.

In $\S 4$ we consider a Poisson-like problem:

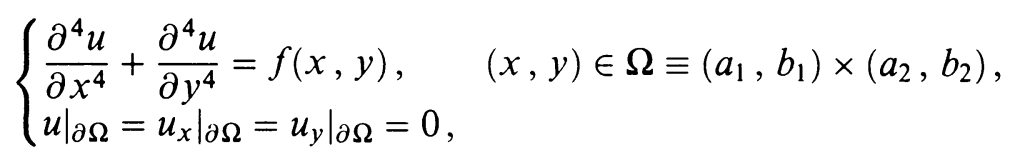

and prove that this kind of problem can also be solved by the same technique described in $§ 3$. Some numerical examples are also given in this section.

\section{SinC APPROXIMATION}

2.1. Notation and background. The goal of this section is to recall notation and definitions of the Sinc function, state some known results, and derive useful formulas that are important for this paper.

First we denote the set of all integers, the set of all real numbers, and the set of all complex numbers by $\mathbf{Z}, \mathbf{R}$, and $\mathbf{C}$, respectively.

Notaition 2.1. (1) $\operatorname{sinc}(z)=\sin (\pi z) / \pi z, \quad z \in \mathbf{Z}$.

Note that $|\operatorname{sinc}(x)| \leq 1$ for any $x \in \mathbf{R}$.

(2) $S(n, h)(z)=\operatorname{sinc}((z-n h) / h), z \in \mathbf{C}, h>0$.

(3) $C(f, h, x)=\sum_{k=-\infty}^{\infty} f(k h) S(k, h)(x), \quad h>0$.

Here, $C(f, h, x)$ is called the Whittaker cardinal function of $f(x)$ whenever this series converges.

(4) $C_{N}(f, h, x)=\sum_{k=-N}^{N} f(k h) S(k, h)(x)$.

Definition 2.1. Let $d>0$, and let $\mathscr{D}_{d}$ denote the region $\{z=x+i y:|y|<d\}$ in the complex plane, and $\phi$ the conformal map of a simply connected domain $\mathscr{D}$ in the complex plane onto $\mathscr{D}_{d}$ such that $\phi(a)=-\infty$ and $\phi(b)=\infty$, where $a$ and $b$ are boundary points of $\mathscr{D}$, i.e., $a, b \in \partial \mathscr{D}$. Let $\psi$ denote the inverse map of $\phi$, and let the arc $\Gamma$, with endpoints $a$ and $b(a, b \notin \Gamma)$, be given by $\Gamma=\psi(-\infty, \infty)$. For $h>0$, let the points $x_{k}$ on $\Gamma$ be given by $x_{k}=\psi(k h), k \in \mathbf{Z}$.

For example, if the finite interval is $(a, b)$, take

$$
\phi(x)=\ln \frac{x-a}{b-x}
$$

and

$$
\psi(w)=\frac{a+b e^{w}}{1+e^{w}}, \quad x_{k}=\frac{a+b e^{k h}}{1+e^{k h}} .
$$

Definition 2.2. Let $B(\mathscr{D})$ denote the family of all functions $f$ that are analytic in $\mathscr{D}$ such that for $x$ real,

$$
\int_{\psi(x+L)}|f(z) d z| \rightarrow 0 \quad(x \rightarrow \infty),
$$

where $L=\{i y:-d \leq y \leq d\}$, and such that

$$
\mathscr{N}(f, \mathscr{D})=\liminf _{C \rightarrow \partial \mathscr{Z}, C \subset \mathscr{Z}} \int_{C}|f(z) d z|<\infty,
$$

where $C$ is a simple closed contour in $\mathscr{D}$. 
The following important theorems were proved by Stenger [7].

Theorem 2.1. If $f \in B(\mathscr{D})$, then

$$
\|f(x)-C(f, h, x)\|_{\infty} \leq \frac{\mathscr{N}(f, \mathscr{D})}{2 \pi d \sinh (\pi d / h)} \leq \frac{2 \mathscr{N}(f, \mathscr{D})}{\pi d} e^{-\pi d / h},
$$

provided $h \leq 2 \pi d / \ln 2$.

Theorem 2.2. Let $f \in B(\mathscr{D})$, and let $f$ satisfy $|f(x)|<C e^{-\alpha|x|}$ for all $x \in \mathbf{R}$, where $C$ and $\alpha$ are positive constants. Then, by choosing $h=\sqrt{\pi d / \alpha N} \leq$ $2 \pi d / \ln 2$, we have

$$
\left\|f(x)-C_{N}(f, h, x)\right\|_{\infty} \leq C_{1} \sqrt{N} \exp (-\sqrt{\pi d \alpha N})
$$

where $C_{1}$ is a constant depending only on $f$ and $\alpha$.

If $x$ is on the curve $\Gamma$, we obtain the following theorem by introducing the conformal map $\phi$.

Theorem 2.3. Let $\phi^{\prime} f \in B(\mathscr{D})$. Then

$$
\begin{aligned}
\mid f(x) & -\sum_{k=-\infty}^{\infty} f\left(x_{k}\right) S(k, h) \circ \phi(x) \mid \leq \frac{\mathscr{N}\left(f \phi^{\prime}, \mathscr{D}\right)}{2 \pi d \sinh (\pi d / h)} \\
\leq & \frac{2 \mathcal{N}\left(f \phi^{\prime}, \mathscr{D}\right)}{\pi d} e^{-\pi d / h}, \quad x \in \Gamma .
\end{aligned}
$$

Moreover, if $|f(x)| \leq C e^{-\alpha|\phi(x)|}, x \in \Gamma$, for some positive constants $C$ and $\alpha$, and if the selection $h=\sqrt{\pi d / \alpha N} \leq 2 \pi d / \ln 2$ is made in (2.3), then

$$
\left|f(x)-\sum_{k=-N}^{N} f\left(x_{k}\right) S(k, h) \circ \phi(x)\right| \leq C_{2} \sqrt{N} \exp (-\sqrt{\pi d \alpha N}), \quad x \in \Gamma,
$$

where $C_{2}$ depends only on $f, d$ and $\alpha$.

2.2. Approximation of derivatives on $\Gamma$. As indicated by Stenger in [9], the formula (2.3) is not useful for accurately approximating derivatives of $f$ on $\Gamma$, except on $\mathbf{R}$, since the terms $\phi^{\prime} S(k, h) \circ \phi$ are unbounded on $\Gamma$. Hence, a "nullifier" function $g$ is introduced to get a formula for approximating $f^{(m)}$ on $\Gamma$.

Let $g$ be an analytic function defined on $\mathscr{D}$, and for $k \in \mathbf{Z}$ set

$$
S_{k}(z)=g(z) \operatorname{sinc}\left[\frac{\phi(z)-k h}{h}\right]=g(z) S(k, h) \circ \phi(z), \quad z \in \mathscr{D} .
$$

The following theorem has been established in [5]. Since part of the proof of this theorem will be used later, the proof is included here.

Theorem 2.4. Let $m$ be a nonnegative integer, and let $\phi^{\prime} f / g \in B(\mathscr{D})$. Let there exist positive constants $\alpha, C_{2}$ depending only on $m, d$, and $g$, a constant $C_{1}$ 
depending only on $m$ and $g$, and a constant $C_{0}$ depending only on $g$ and $f$, such that

$$
\begin{gathered}
\left|\frac{f(x)}{g(x)}\right| \leq C_{0} e^{-\alpha|\phi(x)|}, \quad x \in \Gamma, \\
\left|\left(\frac{d}{d x}\right)^{n} S_{k}(x)\right| \leq C_{1} h^{-n} \quad \forall x \in \Gamma, n=0,1, \ldots, m, \\
\mid\left(\frac{d}{d x}\right)^{n}\left\{\begin{array}{c}
\left.\frac{g(x) \sin [\pi \phi(x) / h]}{\phi(z)-\phi(x)}\right\} \mid \\
\leq C_{2} h^{-n} \quad \forall x \in \Gamma, \quad z \in \partial \mathscr{D}, n=0,1, \ldots, m .
\end{array}\right.
\end{gathered}
$$

Then there exists a constant $K$ depending only on $m, d, \alpha, g$, and $f$ such that if $h=\sqrt{\pi d / \alpha N}$, then

$$
\left|f^{(n)}(x)-\sum_{j=-N}^{N} \frac{f\left(x_{j}\right)}{g\left(x_{j}\right)} S_{j}^{(n)}(x)\right| \leq K N^{\frac{n+1}{2}} \exp (-\sqrt{\pi d \alpha N})
$$

for all $x \in \Gamma$, and for $n=0,1, \ldots, m$, where $\phi\left(x_{j}\right)=j h$.

Proof. It is easy to show from the residual theorem that if $\phi^{\prime} f / g \in B(\mathscr{D})$, then for each $x \in \Gamma$,

$$
\begin{aligned}
f(x) & -\sum_{j=-\infty}^{\infty} \frac{f\left(x_{j}\right)}{g\left(x_{j}\right)} S_{j}(x)=f(x)-\sum_{j=-\infty}^{\infty} \frac{f\left(x_{j}\right)}{g\left(x_{j}\right)} g(x) S(j, h) \circ \phi(x) \\
& =\frac{g(x) \sin [\pi \phi(x) / h]}{2 \pi i} \int_{\partial \mathscr{D}} \frac{\left[f(z) \phi^{\prime}(z) / g(z)\right] d z}{[\phi(z)-\phi(x)] \sin [\pi \phi(z) / h]} .
\end{aligned}
$$

Differentiating both sides of (2.8) $n$ times with respect to $x$, using (2.7), and noting that $|\Im \phi(z)|=d$ for all $z \in \partial \mathscr{D}$ so that $|\sin [\pi \phi(z) / h]| \geq \sinh (\pi d / h)$, we find that

$$
\begin{aligned}
& \left|f^{(n)}(x)-\sum_{j=-\infty}^{\infty} \frac{f\left(x_{j}\right)}{g\left(x_{j}\right)} S_{j}^{(n)}(x)\right| \\
& \quad \leq \frac{1}{2 \pi} \int_{\partial \mathscr{D}}\left|\left(\frac{d}{d x}\right)^{n}\left\{\frac{g(x) \sin [\pi \phi(x) / h]}{\phi(z)-\phi(x)}\right\}\right|\left|\frac{f(z) \phi^{\prime}(z) / g(z)}{\sin [\pi \phi(z) / h]} d z\right| \\
& \quad \leq \frac{C_{2} h^{-n}}{2 \pi \sinh (\pi d / h)} \int_{\partial \mathscr{D}}\left|\frac{f(z) \phi^{\prime}(z)}{g(z)} d z\right| \\
& \quad \leq \frac{2 C_{2} h^{-n}}{\pi} e^{-\pi d / h} \int_{\partial \mathscr{D}}\left|\frac{f(z) \phi^{\prime}(z)}{g(z)} d z\right|,
\end{aligned}
$$

since $1 / \sinh (\pi d / h)<4 e^{-\pi d / h}$ provided $h<2 \pi d / \ln 2$. 
Now from the triangle inequality we have that

$$
\begin{aligned}
& \left|f^{(n)}(x)-\sum_{j=-N}^{N} \frac{f\left(x_{j}\right)}{g\left(x_{j}\right)} S_{j}^{(n)}(x)\right| \\
& \quad \leq\left|f^{(n)}(x)-\sum_{j=-\infty}^{\infty} \frac{f\left(x_{j}\right)}{g\left(x_{j}\right)} S_{j}^{(n)}(x)\right|+\left|\sum_{|j|>N} \frac{f\left(x_{j}\right)}{g\left(x_{j}\right)} S_{j}^{(n)}(x)\right| .
\end{aligned}
$$

Recalling that $\phi\left(x_{j}\right)=j h$ and applying (2.5) and (2.6), we get that

$$
\begin{aligned}
\left|\sum_{|j|>N} \frac{f\left(x_{j}\right)}{g\left(x_{j}\right)} S_{j}^{(n)}(x)\right| & \leq \sum_{|j|>N} C_{0} e^{-\alpha\left|\phi\left(x_{j}\right)\right|} C_{1} h^{-n} \\
& =\frac{2 C_{0} C_{1}}{h^{n}} \sum_{j=N+1}^{\infty} e^{-\alpha j h} \leq \frac{2 C_{0} C_{1}}{\alpha h^{n+1}} e^{-\alpha N h} .
\end{aligned}
$$

Hence, the above inequality with (2.9) gives

$$
\begin{aligned}
& \left|f^{(n)}(x)-\sum_{j=-N}^{N} \frac{f\left(x_{j}\right)}{g\left(x_{j}\right)} S_{j}^{(n)}(x)\right| \\
& \quad \leq \frac{2 C_{2} h^{-n}}{\pi} e^{-\pi d / h} \int_{\partial \mathscr{D}}\left|\frac{f(z) \phi^{\prime}(z)}{g(z)} d z\right|+\frac{2 C_{0} C_{1}}{\alpha h^{n+1}} e^{-\alpha N h} .
\end{aligned}
$$

Since $\phi(\partial \mathscr{D})=\partial \mathscr{D}_{d}$, the integral $\int_{\partial \mathscr{D}}\left|\frac{f(z) \phi^{\prime}(z)}{g(z)} d z\right|$ is a constant depending only on $f, g$, and $d$. The proof is then completed if we set $h=\sqrt{\pi d / \alpha N}$ and $K=\max \left\{K_{n}, n=0,1, \ldots, m\right\}$, where

$$
K_{n}=\frac{2 C_{2}}{\pi} \int_{\partial \mathscr{D}}\left|\frac{f(z) \phi^{\prime}(z)}{g(z)} d z\right|\left(\frac{\alpha}{\pi d}\right)^{\frac{n}{2}}+\frac{2 C_{0} C_{1}}{\alpha}\left(\frac{\alpha}{\pi d}\right)^{\frac{n+1}{2}} .
$$

It should be noted that the function $g$ takes on different forms for different functions $\phi$; usually, $g(x)=\left(\phi^{\prime}(x)\right)^{-\beta}$ is satisfactory, which was verified by Lundin and Stenger in [5] for the cases of approximation over $(-1,1)$ and $(0, \infty)$.

2.3. Extension of the approximation to two dimensions. For the sake of simplicity, we consider in the rest of this section only the two-dimensional case, although the following procedure works for any dimension. We will develop some useful results of the Sinc-collocation approximation for two dimensions.

We set $\mathbf{x}=(x, y)$. Later, a bar over some letter always means that this letter refers to the $y$-coordinate.

Given $\mathbf{d}=\left(d_{1}, d_{2}\right) \in \mathbf{R}^{2}$ with $d_{i}>0$ for $i=1,2$ and conformal maps $\psi: \mathscr{D}_{d_{1}} \rightarrow \mathscr{D}_{1}$ and $\bar{\psi}: \mathscr{D}_{d_{2}} \rightarrow \mathscr{D}_{2}$, set $\mathscr{D}_{\mathbf{d}}=\mathscr{D}_{d_{1}} \times \mathscr{D}_{d_{2}}$ and $\mathscr{D}=\mathscr{D}_{1} \times \mathscr{D}_{2}$, define $\psi: \mathscr{D}_{\mathbf{d}} \rightarrow \mathscr{D}$ in coordinate-wise fashion, so $\underline{\psi}=\psi \times \bar{\psi}$. Set $\Gamma_{1}=\psi(\mathbf{R})$ and $\Gamma_{2}=\bar{\psi}(\mathbf{R})$, and set $\Gamma=\psi\left(\mathbf{R}^{2}\right)=\Gamma_{1} \times \Gamma_{2}$.

Furthermore, set, as usual, $\underline{\phi}=\underline{\psi}^{-1}$. Given $\mathbf{h}=\left(h_{1}, h_{2}\right) \in \mathbf{R}^{2}$ with positive components $h_{i}(i=\overline{1}, 2)$, and given $\mathbf{k} \in \mathbf{Z}^{2}$, define $\left(x_{k_{1}}, y_{k_{2}}\right)=$ 
$\left(\psi\left(k_{1} h_{1}\right), \bar{\psi}\left(k_{2} h_{2}\right)\right)$ and

$$
S_{k}(x)=g(x) S\left(k, h_{1}\right) \phi(x), \quad \bar{S}_{k}(y)=\bar{g}(y) \bar{S}\left(k, h_{2}\right)(y),
$$

where $k \in \mathbf{Z}$.

We will note some analogues of the previous theorems as they apply to the complex functions $F: \mathscr{D} \rightarrow \mathbf{C}$. Let

$$
\mathscr{D}_{1}^{\prime}=\mathscr{D}_{1} \times \Gamma_{2}, \quad \mathscr{D}_{2}^{\prime}=\Gamma_{1} \times \mathscr{D}_{2} .
$$

We say that $F \in B\left(\mathscr{D}_{j}^{\prime}\right)$ if $F \in B\left(\mathscr{D}_{j}\right)$ by fixing the remaining variable.

Analogous to Theorem 2.4, it is not difficult to get

Lemma 2.1. (1) If $f(x, y) \phi^{\prime}(x) / g(x) \in B\left(\mathscr{D}_{1}^{\prime}\right)$, then for any $(x, y) \in \Gamma$, we have

$$
\begin{aligned}
f(x, y) & -\sum_{k=-\infty}^{\infty} \frac{f\left(x_{k}, y\right)}{g\left(x_{k}\right)} S_{k}(x) \\
& =\frac{g(x) \sin \left[\pi \phi(x) / h_{1}\right]}{2 \pi i} \int_{\partial \mathscr{D}_{1}} \frac{f(z, y) \phi^{\prime}(z) / g(z)}{[\phi(z)-\phi(x)] \sin \left[\pi \phi(z) / h_{1}\right]} d z .
\end{aligned}
$$

(2) Let $f(x, y) \phi^{\prime}(x) / g(x) \in B\left(\mathscr{D}_{1}^{\prime}\right)$. Assume

$$
\left|\left(\frac{d}{d x}\right)^{\ell}\left\{\frac{g(x) \sin \left[\pi \phi(x) / h_{1}\right]}{\phi(z)-\phi(x)}\right\}\right| \leq C_{1,2} h_{1}^{-\ell}
$$

for any $x \in \Gamma_{1}, \quad z \in \partial \mathscr{D}_{1}$, and $h_{1}<2 \pi d_{1} / \ln 2$. Then

$$
\begin{aligned}
\mid\left(\frac{d}{d x}\right)^{\ell} & \{f(x, y)\}-\sum_{k=-\infty}^{\infty} \frac{f\left(x_{k}, y\right)}{g\left(x_{k}\right)}\left(\frac{d}{d x}\right)^{\ell}\left\{S_{k}(x)\right\} \mid \\
& \leq \frac{C_{1,2} h_{1}^{-\ell}}{2 \pi \sin \left[\pi d_{1} / h_{1}\right]} \int_{\partial \mathscr{D}_{1}}\left|\frac{f(z, y) \phi^{\prime}(z)}{g(z)} d z\right| \\
& \leq \frac{2 C_{1,2}}{\pi} h_{1}^{-\ell} e^{-\frac{\pi d_{1}}{h_{1}}} \int_{\partial \mathscr{D}_{1}}\left|\frac{f(z, y) \phi^{\prime}(z)}{g(z)} d z\right| .
\end{aligned}
$$

(3) If all the conditions in (2) are satisfied and

$$
\left|\frac{f(x, y)}{g(x)}\right| \leq C_{0} e^{-\alpha_{1}|\phi(x)|} \quad \forall(x, y) \in \Gamma,
$$

and

$$
\left|\left(\frac{d}{d x}\right)^{\ell}\left\{S_{k}(x)\right\}\right| \leq C_{1,1} h_{1}^{-\ell} \quad \forall x \in \Gamma_{1},
$$

then

$$
\begin{aligned}
& \left|\left(\frac{d}{d x}\right)^{\ell} f(x, y)-\sum_{k=-N_{j}}^{N_{j}} \frac{f\left(x_{k}, y\right)}{g\left(x_{k}\right)}\left(\frac{d}{d x}\right)^{\ell}\left\{S_{k}(x)\right\}\right| \\
& \quad \leq \frac{2 C_{1,2}}{\pi} h_{1}^{-\ell} e^{-\frac{\pi d_{1}}{h_{1}}} \int_{\partial \mathscr{C}_{1}}\left|\frac{f(z, y) \phi^{\prime}(z)}{g(z)} d z\right|+\frac{2 C_{0} C_{1,1}}{\alpha_{1} h_{1}^{\ell+1}} e^{-\alpha_{1} N_{1} h_{1}} .
\end{aligned}
$$


A similar formula can be obtained for the $y$-coordinate.

We always assume later $\Gamma_{1}=\left(a_{1}, b_{1}\right)$ and $\Gamma_{2}=\left(a_{2}, b_{2}\right)$ to be finite, so

$$
\begin{array}{lll}
\phi(x)=\ln \frac{x-a_{1}}{b_{1}-x}, & g(x)=\frac{\left(x-a_{1}\right)^{\beta_{1}}\left(b_{1}-x\right)^{\beta_{1}}}{\left(b_{1}-a_{1}\right)^{\beta_{1}}}, & \beta_{1}>0, \\
\bar{\phi}(y)=\ln \frac{y-a_{2}}{b_{2}-y}, & \bar{g}(y)=\frac{\left(y-a_{2}\right)^{\beta_{2}}\left(b_{2}-y\right)^{\beta_{2}}}{\left(b_{2}-a_{2}\right)^{\beta_{2}}}, & \beta_{2}>0 .
\end{array}
$$

It then follows that

$$
\begin{aligned}
& |g(x)| \leq \frac{\left(b_{1}-a_{1}\right)^{\beta_{1}}}{4^{\beta_{1}}} \text { for } a_{1}<x<b_{1}, \\
& |\bar{g}(y)| \leq \frac{\left(b_{2}-a_{2}\right)^{\beta_{2}}}{4^{\beta_{2}}} \text { for } a_{2}<y<b_{2} .
\end{aligned}
$$

Lemma 2.2. Let $\phi(x)$ and $g(x)$ be defined as in (2.12) and $\beta_{1} \geq \ell$, where $\ell$ is a nonnegative integer. If $h_{1}>0$ is uniformly bounded by $h^{*}$, then

$$
\begin{aligned}
\left|S_{k}^{(\ell)}(x)\right| & =\left|\left(\frac{d}{d x}\right)^{\ell}\left\{g(x) S\left(k, h_{1}\right) \circ \phi(x)\right\}\right| \\
& \leq C_{0} h_{1}^{-\ell}\left(x-a_{1}\right)^{\beta_{1}-\ell}\left(b_{1}-x\right)^{\beta_{1}-\ell} \leq K h_{1}^{-\ell} \quad \forall x \in \Gamma_{1},
\end{aligned}
$$

where $C_{0}$ and $K$ depend only on $\beta_{1}, \ell, a_{1}, b_{1}$, and $h^{*}$.

Proof. The proof is almost the same as that of Theorem 6.3 in [5] by a translation argument.

Remark 2.1. It is interesting to note that the constant $K$ does not depend on $k \in \mathbf{Z}$, which means that the condition $\left|S_{0}^{(\ell)}(x)\right| \leq K h_{1}^{-\ell}$ for all $x \in \Gamma_{1}$ implies $\left|S_{k}^{(\ell)}(x)\right| \leq K h_{1}^{-\ell}$ for all $x \in \Gamma_{1}$ and all $k \in \mathbf{Z}$. There is an analogous result for $\left|\bar{S}_{k}^{(\ell)}(y)\right|$.

Lemma 2.3. Let $f(x, y)$ satisfy

(1)

$$
\left|\frac{f(x, y)}{g(x) \bar{g}(y)}\right| \leq C_{0} e^{-\alpha_{1}|\phi(x)|} e^{-\alpha_{2}|\bar{\phi}(y)|}, \quad(x, y) \in \overline{\mathscr{D}},
$$

(2)

$$
\frac{f(x, y) \phi^{\prime}(x)}{g(x)} \in B\left(\mathscr{D}_{1}^{\prime}\right), \quad \frac{f(x, y) \bar{\phi}^{\prime}(y)}{\bar{g}(y)} \in B\left(\mathscr{D}_{2}^{\prime}\right)
$$

(3)

$$
\left|\left(\frac{d}{d x}\right)^{n}\left\{\frac{g(x) \sin \left[\pi \phi(x) / h_{1}\right]}{\phi(z)-\phi(x)}\right\}\right| \leq C_{1} h_{1}^{-n} \quad \text { for all } x \in \Gamma_{1}, \quad z \in \partial \mathscr{D}_{1},
$$

where $C_{1}=C_{1}\left(n, g, a_{1}, b_{1}\right)$, and

$$
\left|\frac{\bar{g}(y) \sin \left[\pi \bar{\phi}(y) / h_{2}\right]}{\bar{\phi}(z)-\bar{\phi}(y)}\right| \leq C_{2} \quad \text { for all } y \in \Gamma_{2}, \quad z \in \partial \mathscr{D}_{2},
$$


where $C_{2}=C_{2}\left(\bar{g}, a_{2}, b_{2}\right)$,

(4)

$$
\left|S_{0}^{(n)}(x)\right| \leq C_{4} h_{1}^{-n} \quad \forall x \in \Gamma_{1}, \quad\left|\bar{S}_{0}(y)\right| \leq C_{5} \quad \forall y \in \Gamma_{2},
$$

where $C_{4}=C_{4}\left(\beta_{1}, n, a_{1}, b_{1}, h^{*}\right)$ and $C_{5}=C_{5}\left(\beta_{2}, a_{2}, b_{2}, h^{*}\right)$.

Let $h_{j}=\sqrt{\pi d_{j} / \alpha_{j} N_{j}} \leq h^{*}, j=1,2$. Then there exists a constant $C$ depending on $g, \bar{g}, n, \beta_{j}, a_{j}$, and $b_{j}(j=1,2)$ such that

$$
\begin{gathered}
\left|\left(\frac{d}{d x}\right)^{n} f(x, y)-\sum_{k_{1}=-N_{1}}^{N_{1}} \sum_{k_{2}=-N_{2}}^{N_{2}} \frac{f_{k_{1} k_{2}}}{g_{k_{1}} \bar{g}_{k_{2}}} S_{k_{1}}^{(n)}(x) \bar{S}_{k_{2}}(y)\right| \\
\leq C N_{1}^{\frac{n+1}{2}}\left(E_{1}|\bar{g}(y)| e^{-\alpha_{2}|\bar{\phi}(y)|}+N_{2}^{\frac{1}{2}} E_{2}\right),
\end{gathered}
$$

where $f_{k_{1} k_{2}}=f\left(x_{k_{1}}, y_{k_{2}}\right), g_{k_{1}}=g\left(x_{k_{1}}\right), \bar{g}_{k_{2}}=\bar{g}\left(y_{k_{2}}\right)$, and $E_{j}=e^{-\sqrt{\pi d_{j} \alpha_{j} N_{j}}}$. Proof. We can get

$$
\left|\left(\frac{d}{d x}\right)^{n} f(x, y)-\sum_{k_{1}=-N_{1}}^{N_{1}} \sum_{k_{2}=-N_{2}}^{N_{2}} \frac{f_{k_{1} k_{2}}}{g_{k_{1}} \bar{g}_{k_{2}}} S_{k_{1}}^{(n)}(x) \bar{S}_{k_{2}}(y)\right| \leq J_{1}+J_{2},
$$

where

$$
\begin{gathered}
J_{1}=\left|\left(\frac{d}{d x}\right)^{n} f(x, y)-\sum_{k_{1}=-N_{1}}^{N_{1}} \frac{f\left(x_{k_{1}}, y\right)}{g_{k_{1}}} S_{k_{1}}^{(n)}(x)\right|, \\
J_{2}=\mid \sum_{k_{1}=-N_{1}}^{N_{1}} \frac{S_{k_{1}}^{(n)}(x)}{g_{k_{1}}}\left[f\left(x_{k_{1}}, y\right)-\sum_{k_{2}=-N_{2}}^{N_{2}} \frac{f\left(x_{k_{1}}, y_{k_{2}}\right)}{\bar{g}_{k_{2}}} \bar{S}_{k_{2}}(y)\right] .
\end{gathered}
$$

Using Lemma 2.1(3), we have

$$
\begin{aligned}
& J_{1} \leq \frac{2 C_{1}}{\pi} h_{1}^{-n} e^{-\frac{\pi d_{1}}{h_{1}}} \int_{\partial \mathscr{D}_{1}}\left|\frac{f(x, y) \phi^{\prime}(x)}{g(x)} d x\right| \\
& +\frac{2 C_{0} C_{4}|\bar{g}(y)| e^{-\alpha_{2}|\bar{\phi}(y)|}}{\alpha_{1} h_{1}^{n+1}} e^{-\alpha_{1} N_{1} h_{1}} \\
& \leq \frac{2 C_{0} C_{1}}{\pi} h_{1}^{-n} e^{-\frac{\pi d_{1}}{h_{1}}}|\bar{g}(y)| e^{-\alpha_{2}|\bar{\phi}(y)|} \int_{\partial \mathscr{D}_{1}}\left|e^{-\alpha_{1}|\phi(x)|} \phi^{\prime}(x) d x\right| \\
& +\frac{2 C_{0} C_{4}}{\alpha_{1} h_{1}^{n+1}}|\bar{g}(y)| e^{-\alpha_{2}|\bar{\phi}(y)|} e^{-\alpha_{1} N_{1} h_{1}} \\
& \leq K_{1}|\bar{g}(y)| e^{-\alpha_{2}|\bar{\phi}(y)|} N_{1}^{\frac{n+1}{2}} e^{-\sqrt{\pi d_{1} \alpha_{1} N_{1}}},
\end{aligned}
$$

where the constant $K_{1}$ depends only on $n, g, a_{1}, b_{1}, d_{1}, \alpha_{1}$, and $h^{*}$.

Using Lemma 2.1(3) again, we get

$$
\begin{aligned}
& \left|f\left(x_{k_{1}}, y\right)-\sum_{k_{2}=-N_{2}}^{N_{2}} \frac{f\left(x_{k_{1}}, y_{k_{2}}\right)}{\bar{g}_{k_{2}}} \bar{S}_{k_{2}}(y)\right| \\
& \quad \leq \frac{2 C_{0}}{\alpha_{2}} \frac{C_{5}}{h_{2}}\left|g\left(x_{k_{1}}\right)\right| e^{-\alpha_{1}\left|\phi\left(x_{k_{1}}\right)\right|} e^{-\alpha_{2} N_{2} h_{2}}+\frac{2 C_{2}}{\pi} e^{-\frac{\pi d_{2}}{h_{2}}} \int_{\partial \mathscr{D}_{2}}\left|\frac{f\left(x_{k_{1}}, y\right) \bar{\phi}^{\prime}(y)}{\bar{g}(y)} d y\right| \\
& \leq K_{2}^{\prime}\left|g\left(x_{k_{1}}\right)\right| e^{-\alpha_{1}\left|\phi\left(x_{k_{1}}\right)\right|} N_{2}^{\frac{1}{2}} e^{-\sqrt{\pi d_{2} \alpha_{2} N_{2}}}
\end{aligned}
$$


where the constant $K_{2}^{\prime}$ depends only on $\bar{g}, a_{2}, b_{2}, d_{2}, \alpha_{2}$, and $h^{*}$. Hence,

$$
J_{2} \leq\left(\sum_{k_{1}=-N_{1}}^{N_{1}}\left|S_{k_{1}}^{(n)}(x)\right| e^{-\alpha_{1}\left|\phi\left(x_{k_{1}}\right)\right|}\right) K_{2}^{\prime} N_{2}^{\frac{1}{2}} e^{-\sqrt{\pi d_{2} \alpha_{2} N_{2}}} .
$$

Noting condition (4), and recalling Remark 2.1, we know that

$$
\left|S_{k_{1}}^{(n)}(x)\right| \leq C_{4} h_{1}^{-n} \text { for any } k_{1} \in \mathbf{Z} .
$$

Therefore,

$$
\begin{aligned}
& \sum_{k_{1}=-N_{1}}^{N_{1}}\left|S_{k_{1}}^{(n)}(x)\right| e^{-\alpha_{1}\left|\phi\left(x_{k_{1}}\right)\right|} \leq C_{4} h_{1}^{-n} \sum_{k_{1}=-N_{1}}^{N_{1}} e^{-\alpha_{1}\left|\phi\left(x_{k_{1}}\right)\right|} \\
& \quad=C_{4} h_{1}^{-n} \sum_{k_{1}=-N_{1}}^{N_{1}} e^{-\alpha_{1}\left|k_{1}\right| h_{1}} \leq C_{4} h_{1}^{-n}\left[1+2 \frac{e^{-\alpha_{1} h_{1}}\left(1-e^{-\alpha_{1} N_{1} h_{1}}\right)}{1-e^{-\alpha_{1} h_{1}}}\right] \\
& \quad \leq C_{4} h_{1}^{-n}\left(1+\frac{2}{\alpha_{1} h_{1}}\right) \leq C_{4}^{\prime} h_{1}^{-(n+1)} .
\end{aligned}
$$

Thus, we obtain

$$
J_{2} \leq K_{2} N_{1}^{\frac{(n+1)}{2}} N_{2}^{\frac{1}{2}} e^{-\sqrt{\pi d_{2} \alpha_{2} N_{2}}}
$$

where $K_{2}$ depends only on $n, g, \bar{g}, a_{j}, b_{j}, d_{j}, \alpha_{j}(j=1,2)$, and $h^{*}$.

Combining (2.16) and (2.17) completes the proof.

In the following we denote $\left(\partial^{n_{1}+n_{2}} / \partial x^{n_{1}} \partial y^{n_{2}}\right) f(x, y)$ by $f^{\left(n_{1}, n_{2}\right)}(x, y)$.

Theorem 2.5. Let $f(x, y)$ satisfy

(1)

$$
\begin{gathered}
\left|\frac{f^{\left(0, n_{2}\right)}(x, y)}{g(x) \bar{g}(y)}\right| \leq C_{0} e^{-\alpha_{1}|\phi(x)|} e^{-\alpha_{2}|\bar{\phi}(y)|} \quad \forall(x, y) \in \overline{\mathscr{D}}, \\
\left|\frac{f(x, y)}{g(x) \bar{g}(y)}\right| \leq C_{0} e^{-\alpha_{1}|\phi(x)|} e^{-\alpha_{2}|\bar{\phi}(y)|} \quad \forall(x, y) \in \overline{\mathscr{D}},
\end{gathered}
$$

$$
\frac{f^{\left(0, n_{2}\right)}(x, y) \phi^{\prime}(x)}{g(x)} \in B\left(\mathscr{D}_{1}^{\prime}\right), \quad \frac{f(x, y) \bar{\phi}^{\prime}(y)}{\bar{g}(y)} \in B\left(\mathscr{D}_{2}^{\prime}\right),
$$

$$
\left|\left(\frac{d}{d x}\right)^{n_{1}}\left\{\frac{g(x) \sin \left[\pi \phi(x) / h_{1}\right]}{\phi(z)-\phi(x)}\right\}\right| \leq C_{1} h_{1}^{-n_{1}}
$$

for all $x \in \Gamma_{1}, z \in \partial \mathscr{D}_{1}$, where $C_{1}=C_{1}\left(n_{1}, g, a_{1}, b_{1}\right)$, and

$$
\left|\left(\frac{d}{d y}\right)^{n_{2}}\left\{\frac{\bar{g}(y) \sin \left[\pi \bar{\phi}(y) / h_{2}\right]}{\bar{\phi}(z)-\bar{\phi}(y)}\right\}\right| \leq C_{2} h_{2}^{-n_{2}}
$$


for all $y \in \Gamma_{2}, z \in \partial \mathscr{D}_{2}$, where $C_{2}=C_{2}\left(n_{2}, \bar{g}, a_{2}, b_{2}\right)$,

(4)

$$
\left|S_{0}^{\left(n_{1}\right)}(x)\right| \leq C_{3} h_{1}^{-n_{1}} \quad \forall x \in \Gamma_{1}, \quad\left|\bar{S}_{0}^{\left(n_{2}\right)}(y)\right| \leq C_{4} h_{2}^{-n_{2}} \quad \forall y \in \Gamma_{2},
$$

where $C_{j}(j=3,4)$ depend only on $\beta_{j}, n_{j}, a_{j}, b_{j}$, and $h^{*}$.

Let $h_{j}=\sqrt{\pi d_{j} / \alpha_{j} N_{j}} \leq h^{*}, j=1,2$. Then there exists a constant $C$ depending on $g, \bar{g}, n_{j}, \beta_{j}, a_{j}$, and $b_{j}(j=1,2)$ such that

$$
\begin{gathered}
\left|f^{\left(n_{1}, n_{2}\right)}(x, y)-\sum_{k_{1}=-N_{1}}^{N_{1}} \sum_{k_{2}=-N_{2}}^{N_{2}} \frac{f_{k_{1} k_{2}}}{g_{k_{1}} \bar{g}_{k_{2}}} S_{k_{1}}^{\left(n_{1}\right)}(x) \bar{S}_{k_{2}}^{\left(n_{2}\right)}(y)\right| \\
\leq C N_{1}^{\frac{n_{1}+1}{2}}\left(E_{1}|\bar{g}(y)| e^{-\alpha_{2}|\bar{\phi}(y)|}+N_{2}^{\frac{n_{2}+1}{2}} E_{2}\right),
\end{gathered}
$$

where $E_{j}(j=1,2)$ are defined as in Lemma 2.3.

Proof. First we have

$$
\left|f^{\left(n_{1}, n_{2}\right)}(x, y)-\sum_{k_{1}=-N_{1}}^{N_{1}} \sum_{k_{2}=-N_{2}}^{N_{2}} \frac{f_{k_{1} k_{2}}}{g_{k_{1}} \bar{g}_{k_{2}}} S_{k_{1}}^{\left(n_{1}\right)}(x) \bar{S}_{k_{2}}^{\left(n_{2}\right)}(y)\right| \leq H_{1}+H_{2},
$$

where

$$
\begin{gathered}
H_{1}=\left|f^{\left(n_{1}, n_{2}\right)}(x, y)-\sum_{k_{1}=-N_{1}}^{N_{1}} \frac{f^{\left(0, n_{2}\right)}\left(x_{k_{1}}, y\right)}{g_{k_{1}}} S_{k_{1}}^{\left(n_{1}\right)}(x)\right|, \\
H_{2}=\left|\sum_{k_{1}=-N_{1}}^{N_{1}} \frac{S_{k_{1}}^{\left(n_{1}\right)}(x)}{g_{k_{1}}}\left[f^{\left(0, n_{2}\right)}\left(x_{k_{1}}, y\right)-\sum_{k_{2}=-N_{2}}^{N_{2}} \frac{f\left(x_{k_{1}}, y_{k_{2}}\right)}{\bar{g}_{k_{2}}} \bar{S}_{k_{2}}(y)\right]\right| .
\end{gathered}
$$

Using Lemma 2.1 and Lemma 2.3, we get

$$
H_{1} \leq K_{1}|\bar{g}(y)| e^{-\alpha_{2}|\bar{\phi}(y)|} N_{1}^{\frac{n_{1}+1}{2}} e^{-\sqrt{\pi d_{1} \alpha_{1} N_{1}}} .
$$

Using Lemma 2.1(3) again, we then obtain

$$
\begin{array}{r}
\left|f^{\left(0, n_{2}\right)}\left(x_{k_{1}}, y\right)-\sum_{k_{2}=-N_{2}}^{N_{2}} \frac{f\left(x_{k_{1}}, y_{k_{2}}\right)}{\bar{g}_{k_{2}}} \bar{S}_{k_{2}}^{\left(n_{2}\right)}(y)\right| \\
\leq K_{2}^{\prime}\left|g\left(x_{k_{1}}\right)\right| e^{-\alpha_{1}\left|\phi\left(x_{k_{1}}\right)\right|} N_{2}^{\frac{n_{2}+1}{2}} e^{-\sqrt{\pi d_{2} \alpha_{2} N_{2}}},
\end{array}
$$

where the constant $K_{2}^{\prime}$ depends only on $\bar{g}, a_{2}, b_{2}, d_{2}, \alpha_{2}$, and $h^{*}$. Hence,

$$
H_{2} \leq K_{2} N_{1}^{\frac{\left(n_{1}+1\right)}{2}} N_{2}^{\frac{\left(n_{2}+1\right)}{2}} e^{-\sqrt{\pi d_{2} \alpha_{2} N_{2}}},
$$

where $K_{2}$ depends only on $n_{j}, g, \bar{g}, a_{j}, b_{j}, d_{j}, \alpha_{j}(j=1,2)$, and $h^{*}$. The proof is completed.

Remark 2.2. In condition $(1), f^{\left(0, n_{2}\right)}$ can be replaced by $f^{\left(n_{1}, 0\right)}$.

Recalling (2.14) and (2.15), we can improve the result of Theorem 2.5 and get 
Theorem 2.6. Suppose all the conditions in Theorem 2.5 are satisfied. Then

$$
\begin{aligned}
& \left|f^{\left(n_{1}, n_{2}\right)}(x, y)-\sum_{k_{1}=-N_{1}}^{N_{1}} \sum_{k_{2}=-N_{2}}^{N_{2}} \frac{f_{k_{1} k_{2}}}{g_{k_{1}} \bar{g}_{k_{2}}} S_{k_{1}}^{\left(n_{1}\right)}(x) \bar{S}_{k_{2}}^{\left(n_{2}\right)}(y)\right| \\
& \quad \leq C N_{1}^{\frac{n_{1}+1}{2}}\left(E_{1}+N_{2}^{\frac{n_{2}+1}{2}} E_{2}\right) .
\end{aligned}
$$

Moreover, if we let

$$
N_{j}=N, \quad d_{j}=d, \quad \alpha_{j}=\alpha \quad(j=1,2),
$$

then

$$
\begin{aligned}
& \left|f^{\left(n_{1}, n_{2}\right)}(x, y)-\sum_{k_{1}=-N_{1}}^{N_{1}} \sum_{k_{2}=-N_{2}}^{N_{2}} \frac{f_{k_{1} k_{2}}}{g_{k_{1}} \bar{g}_{k_{2}}} S_{k_{1}}^{\left(n_{1}\right)}(x) \bar{S}_{k_{2}}^{\left(n_{2}\right)}(y)\right| \\
& \leq C N^{\frac{n_{1}+n_{2}+2}{2}} \exp (-\sqrt{\pi d \alpha N}) \leq C_{1} \exp \left(-C_{2} \sqrt{N}\right),
\end{aligned}
$$

where $C, C_{1}$, and $C_{2}$ are independent of $h$ and $N$.

Note that the last inequality comes from the well-known formula [1, p. 278]

$$
t^{k} e^{-t} \leq C(\theta) e^{-\theta t}, \quad \theta \in(0,1), t \geq 0 .
$$

It should be noted that when we take $\phi, g$ as in (2.12) and $\bar{\phi}, \bar{g}$ as in (2.13), conditions (2)-(4) in Theorem 2.5 or in Theorem 2.6 can be proved to be automatically satisfied, and that the results in these theorems can be improved when $n_{1}=n_{2}=0$ [11, Theorem 6.5.2].

\section{Solution of $P P$}

3.1. Numerical scheme and the Sylvester equation. Several authors have used Sinc methods to solve the singular Poisson problem $P P$; for instance, Stenger [9, 10] applied the Sinc-Galerkin method to $P P$ and got a linear system, and Lund et al. $[3,4]$ got a symmetric linear system by choosing a suitable weight function. The same exponential convergence rate was obtained in all their papers.

Here we use the Sinc-collocation method to solve $P P$. First, for some nonnegative integer $k$, we set

$$
\delta_{j \ell}^{(k)}=\left.h^{k} \frac{d^{k}}{d \phi^{k}}[S(j, h) \circ \phi(z)]\right|_{z=x_{f}} .
$$

By the well-known formula

$$
S(k, h) \circ \phi(x)=\frac{1}{2} \int_{-1}^{1} e^{i \frac{\pi}{h}[\phi(x)-k h] t} d t,
$$

it is easy to verify that

$$
\delta_{j \ell}^{(0)}= \begin{cases}1 & \text { if } j=\ell, \\ 0 & \text { if } j \neq \ell\end{cases}
$$




$$
\delta_{j \ell}^{(1)}= \begin{cases}0 & \text { if } j=\ell, \\ -\frac{(-1)^{j-\ell}}{j-\ell} & \text { if } j \neq \ell,\end{cases}
$$

$$
\delta_{j \ell}^{(2)}= \begin{cases}-\frac{\pi^{2}}{3} & \text { if } j=\ell, \\ -\frac{2(-1)^{j-\ell}}{(j-\ell)^{2}} & \text { if } j \neq \ell,\end{cases}
$$

$$
\delta_{j \ell}^{(3)}= \begin{cases}0 & \text { if } j=\ell, \\ -\frac{(-1)^{j-\ell}}{(j-\ell)^{3}}\left[6-\pi^{2}(j-\ell)^{2}\right] & \text { if } j \neq \ell,\end{cases}
$$

and

$$
\delta_{j \ell}^{(4)}= \begin{cases}\frac{\pi^{4}}{5} & \text { if } j=\ell, \\ -\frac{(-1)^{j-\ell}}{(j-\ell)^{4}}\left[24-4 \pi^{2}(j-\ell)^{2}\right] & \text { if } j \neq \ell .\end{cases}
$$

Now let

$$
v_{N}(x, y)=\sum_{k_{1}=-N_{1}}^{N_{1}} \sum_{k_{2}=-N_{2}}^{N_{2}} \frac{v_{k_{1} k_{2}}}{g_{k_{1}} \bar{g}_{k_{2}}} S_{k_{1}}(x) \bar{S}_{k_{2}}(y) .
$$

The coefficients $v_{k_{1} k_{2}}\left(\left|k_{1}\right| \leq N_{1},\left|k_{2}\right| \leq N_{2}\right)$ are determined by requiring that

$$
\left.\nabla^{2} v_{N}(x, y)\right|_{x=x_{i_{1}}, y=y_{i_{2}}}=f\left(x_{i_{1}}, y_{i_{2}}\right),
$$

where $\left|i_{1}\right| \leq N_{1},\left|i_{2}\right| \leq N_{2}$.

Plugging (3.8) into (3.9) gives

$$
\begin{gathered}
\sum_{\substack{k_{1}=-N_{1} \\
N_{1}}}^{N_{2}=-N_{2}} \frac{v_{k_{1}} k_{2}}{g_{k_{1}} \bar{g}_{k_{2}}}\left(S_{k_{1}}^{\prime \prime}\left(x_{i_{1}}\right) \bar{S}_{k_{2}}\left(y_{i_{2}}\right)+S_{k_{1}}\left(x_{i_{1}}\right) \bar{S}_{k_{2}}^{\prime \prime}\left(y_{i_{2}}\right)\right) \\
=f_{i_{1} i_{2}}=f\left(x_{i_{1}}, y_{i_{2}}\right), \quad\left|i_{1}\right| \leq N_{1}, \quad\left|i_{2}\right| \leq N_{2} .
\end{gathered}
$$

Note that (3.10) can be written in matrix-vector form as

$$
\mathscr{A} \underline{v}=\underline{\mathscr{F}},
$$

where

$$
\mathscr{A}=\left(\frac{S_{k_{1}}^{\prime \prime}\left(x_{i_{1}}\right) \bar{S}_{k_{2}}\left(y_{i_{2}}\right)+S_{k_{1}}\left(x_{i_{1}}\right) \bar{S}_{k_{2}}^{\prime \prime}\left(y_{i_{2}}\right)}{g_{k_{1}} \bar{g}_{k_{2}}}\right)
$$

and where in the vector $\underline{v}=\left(v_{k_{1} k_{2}},\left|k_{1}\right| \leq N_{1},\left|k_{2}\right| \leq N_{2}\right)$ the natural ordering of gridpoints from left to right, bottom to top is used, and similarly for the vector $\mathscr{F}=\left(f_{i_{1} i_{2}}\right)$. As indicated in [4], the structure of the matrix $\mathscr{A}$ in the equation (3.11) is inherently vectorizable. We now use another procedure to solve this equation. 
It follows from (3.3) that

$$
\frac{S_{k_{1}}\left(x_{i_{1}}\right)}{g\left(x_{k_{1}}\right)}=\delta_{k_{1} i_{1}}^{(0)} \quad \text { and } \quad \frac{\bar{S}_{k_{2}}\left(y_{i_{2}}\right)}{\bar{g}\left(y_{k_{2}}\right)}=\delta_{k_{2} i_{2}}^{(0)},
$$

so (3.10) becomes

$$
\sum_{k_{1}=-N_{1}}^{N_{1}} \frac{v_{k_{1} i_{2}}}{g_{k_{1}}} S_{k_{1}}^{\prime \prime}\left(x_{i_{1}}\right)+\sum_{k_{2}=-N_{2}}^{N_{2}} \frac{v_{i_{1} k_{2}}}{\bar{g}_{k_{2}}} \bar{S}_{k_{2}}^{\prime \prime}\left(y_{i_{2}}\right)=f_{i_{1} i_{2}} .
$$

Let $D_{m}(g)$ denote the $m \times m$ (where $m=2 N+1$ ) diagonal matrix with diagonal elements $g\left(x_{j}\right)(j=-N, \ldots, N)$. Set $\tilde{N}_{1}=2 N_{1}+1$ and $\tilde{N}_{2}=$ $2 N_{2}+1$; then it is not difficult to get the Sylvester equation

$$
S_{x} D_{\tilde{N}_{1}}\left(\frac{1}{g(x)}\right) U+U D_{\tilde{N}_{2}}\left(\frac{1}{\bar{g}(y)}\right) S_{y}^{t}=W
$$

where $S_{x}$ is the $\tilde{N}_{1} \times \tilde{N}_{1}$ matrix whose $(i k)$ th entries are $S_{k}^{\prime \prime}\left(x_{i}\right)$, and $S_{y}$ is the $\tilde{N}_{2} \times \tilde{N}_{2}$ matrix whose $(i k)$ th entries are $\bar{S}_{k}^{\prime \prime}\left(y_{i}\right)$, respectively. The matrices $U$ and $W$ are $\tilde{N}_{1} \times \tilde{N}_{2}$ with $(i k)$ th entries equal to $v_{i k}$ and $f_{i k}$, respectively.

Usually, as recommended by Stenger [8] and carried out by Bowers and Lund $[2,4]$, a procedure to solve $(3.14)$ is as follows. First we choose $g=\phi^{-2}$ and $\bar{g}=\bar{\phi}^{-2} \quad\left(g=\phi^{-1}\right.$ and $\bar{g}=\bar{\phi}^{-1}$ are chosen in the Sinc-Galerkin method). We know that there exist two nonsingular matrices $E_{\tilde{N}_{1} \times \tilde{N}_{1}}$ and $\bar{E}_{\tilde{N}_{2} \times \tilde{N}_{2}}$ which diagonalize $S_{x} D_{\tilde{N}_{1}}\left(\frac{1}{g(x)}\right)$ and $D_{\tilde{N}_{2}}\left(\frac{1}{g(y)}\right) S_{y}^{t}$, respectively, i.e.,

$$
S_{x} D_{\tilde{N}_{1}}\left(\frac{1}{g(x)}\right)=E^{-1} \Lambda E, \quad D_{\tilde{N}_{2}}\left(\frac{1}{\bar{g}(y)}\right) S_{y}^{t}=\bar{E}^{-1} \Lambda^{\prime} \bar{E},
$$

where $\Lambda=\left(\lambda_{i}\right)_{\tilde{N}_{1} \times \tilde{N}_{1}}$ and $\Lambda^{\prime}=\left(\lambda_{i}^{\prime}\right)_{\tilde{N}_{2} \times \tilde{N}_{2}}$ are the diagonal matrices whose diagonal entries are eigenvalues of $S_{x} D_{\tilde{N}_{1}}\left(\frac{1}{g(x)}\right)$ and $D_{\tilde{N}_{2}}\left(\frac{1}{g(y)}\right) S_{y}^{t}$, respectively. Hence, it is easy to get from (3.14) that $\Lambda Y+Y \Lambda^{\prime}=X$, where $Y=\left(y_{i k}\right)_{\tilde{N}_{1} \times \tilde{N}_{2}}=$ $E U \bar{E}^{-1}$ and $X=\left(x_{i k}\right)_{\tilde{N}_{1} \times \tilde{N}_{2}}=E W \bar{E}^{-1}$. The solution is then

$$
Y=\left(\frac{x_{i k}}{\lambda_{i}+\lambda_{k}^{\prime}}\right)
$$

This procedure is preferable in view of storage considerations and the ability to handle very singular problems. But it is not very economical computationally since the inverses of $E$ and $\bar{E}$, or some linear systems, need to be computed in order to get $X, Y$, and $U$, and also, theoretically, there is a potential difficulty in (3.15) if $\lambda_{i}+\lambda_{k}^{\prime}=0$.

3.2. Orthogonalization method. Here we solve (3.14) by an orthogonalization technique which is computationally much more economical although in theory applicable to a more restrictive class of problems (with respect to the singular behavior of the problems) than the above procedure. 
Let $s_{i k}=S_{k}^{\prime \prime}\left(x_{i}\right)$. The relation (2.4) yields

$$
S_{k}^{\prime \prime}\left(x_{i}\right)=g_{i}^{\prime \prime} \delta_{k i}^{(0)}+\left(2 g_{i}^{\prime} \phi_{i}^{\prime}+g_{i} \phi_{i}^{\prime \prime}\right) \frac{\delta_{k i}^{(1)}}{h_{1}}+g_{i} \phi_{i}^{\prime 2} \frac{\delta_{k i}^{(2)}}{h_{1}^{2}} .
$$

Taking $g=\phi^{\prime-\frac{1}{2}}$ and using the identity [4]

$$
\phi^{\prime^{-\frac{3}{2}}}\left(\phi^{\prime^{-\frac{1}{2}}}\right)^{\prime \prime}=-\frac{1}{4},
$$

we get

$$
s_{i k}=\frac{1}{h_{1}^{2}} \phi_{i}^{\frac{3}{2}}\left(\delta_{k i}^{(2)}-\frac{1}{4} h_{1}^{2} \delta_{k i}^{(0)}\right) .
$$

If the choice $\bar{g}=\bar{\phi}^{\prime-\frac{1}{2}}$ is made, we can also write

$$
\bar{s}_{i k}=\bar{S}_{k}^{\prime \prime}\left(y_{i}\right)=\frac{1}{h_{2}^{2}} \bar{\phi}_{i}^{\prime \frac{3}{2}}\left(\delta_{k i}^{(2)}-\frac{1}{4} h_{2}^{2} \delta_{k i}^{(0)}\right) .
$$

Now we let $I_{m}^{(k)}$ be the $m \times m$ matrices $(m=2 N+1)$ whose $(j l)$ th entry is $\delta_{j l}^{(k)}$, given by (3.3)-(3.7). Then the equation (3.14) takes the form

$$
A_{x} Y+Y A_{y}=h^{2} W^{\prime},
$$

where

$$
\begin{gathered}
A_{x}=D_{\tilde{N}_{1}}\left(\phi^{\prime}(x)\right) A_{1} D_{\tilde{N}_{1}}\left(\phi^{\prime}(x)\right), \\
A_{y}=D_{\tilde{N}_{2}}\left(\bar{\phi}^{\prime}(y)\right) A_{2} D_{\tilde{N}_{2}}\left(\bar{\phi}^{\prime}(y)\right), \\
Y=D_{\tilde{N}_{1}}\left(\phi^{\prime-\frac{1}{2}}(x)\right) U D_{\tilde{N}_{2}}\left(\bar{\phi}^{\prime-\frac{1}{2}}(y)\right), \\
W^{\prime}=D_{\tilde{N}_{1}}\left(\phi^{\prime-\frac{1}{2}}(x)\right) W D_{\tilde{N}_{2}}\left(\bar{\phi}^{\prime}-\frac{1}{2}(y)\right),
\end{gathered}
$$

and

$$
A_{j}=\left(a_{i k}\right)_{\tilde{N}_{j} \times \tilde{N}_{j}}=\left(\delta_{k i}^{(2)}-\frac{1}{4} h_{j}^{2} \delta_{k i}^{(0)}\right)=I_{\tilde{N}_{j}}^{(2)}-\frac{1}{4} h_{j}^{2} I_{\tilde{N}_{j}}^{(0)}, \quad j=1,2 .
$$

It should be pointed out that the system (3.18) is identical with the system obtained by using an entirely different procedure (the weighted Sinc-Galerkin method) [4]. There are few classes of basis functions where the collocation method and the Galerkin method yield the same discrete system. Typically, the former is advertised as the one with the simpler assembly, while the latter is usually more accurate. Here, the Sinc function gives the best of both worlds.

Lemma 3.1. Both matrices $A_{x}$ and $A_{y}$ are symmetric negative definite.

The proof of this lemma is easy if we notice that the $A_{j}$, given by (3.21), are symmetric negative definite [8] and the diagonal matrices $D_{\tilde{N}_{1}}\left(\phi^{\prime}(x)\right)$ and $D_{\tilde{N}_{2}}\left(\bar{\phi}^{\prime}(y)\right)$ are nonsingular.

Lemma 3.2. If $h_{1}=h_{2}=h$ and $N_{1}=N_{2}=N$, then $A_{y}=\kappa^{2} A_{x}$, where $\kappa=\left(b_{1}-a_{1}\right) /\left(b_{2}-a_{2}\right)$. 
Proof. It is easy to show from (2.12) and (2.13) that

$$
\phi_{i}^{\prime}=\frac{\left(1+e^{i h}\right)^{2}}{\left(b_{1}-a_{1}\right) e^{i h}} \text { and } \bar{\phi}_{i}^{\prime}=\frac{\left(1+e^{i h}\right)^{2}}{\left(b_{2}-a_{2}\right) e^{i h}} .
$$

Thus, $D_{N}\left(\bar{\phi}^{\prime}(y)\right)=\kappa D_{N}\left(\phi^{\prime}(x)\right)$. The relationships $N_{1}=N_{2}=N$ yield $A_{1}=$ $A_{2}=A$. The proof is then completed by using the formulas (3.19).

From now on, we shall choose $h_{1}=h_{2}=h$ and $N_{1}=N_{2}=N$. Thus, all of the matrices in (3.18) are $m \times m(m=2 N+1)$, so the subscripts on the matrices are omitted in the rest of the paper.

The symmetry of the matrix $A_{x}$ implies that there exists an orthogonal matrix $Q$ with the property that $A_{x}=Q \Lambda Q^{t}$, where $\Lambda=\left(\lambda_{i}\right)$ is the diagonal matrix with the eigenvalues of $A_{x}$ as diagonal entries. It is obvious that $A_{y}=\kappa^{2} Q \Lambda Q^{t}$. Hence, (3.18) becomes

$$
\Lambda Y^{\prime}+\kappa^{2} Y^{\prime} \Lambda=h^{2} Z
$$

where $Y^{\prime}=\left(y_{i k}^{\prime}\right)=Q^{t} Y Q$ and $Z=\left(z_{i k}\right)=Q^{t} W^{\prime} Q$. The solution of (3.22) is then

$$
Y^{\prime}=\left(y_{i k}^{\prime}\right)=\left(\frac{h^{2} z_{i k}}{\lambda_{i}+\kappa^{2} \lambda_{k}}\right) .
$$

Lemma 3.1 implies $\lambda_{i}+\kappa^{2} \lambda_{k}<0$, so the potential difficulty appearing in (3.15) is avoided.

To calculate the original $U$ from $D\left(\phi^{\prime \frac{1}{2}}(x)\right) Q Y^{\prime} Q^{t} D\left(\bar{\phi}^{\prime \frac{1}{2}}(y)\right)$ requires only matrix-matrix products, which can be easily done in parallel.

3.3. Computational results. The Sinc-collocation method with orthogonalization has been tested on a large family of problems, both analytic and singular. As is indicated in [2], one of the advantages of the Sinc method is that it automatically determines the graded mesh. So there is no modification in the discrete system (3.18) when it passes from analytic problems to singular problems. All the following three examples are singular, and the second derivatives of their true solutions are undefined on the boundary.

The discussion in the previous section leads us to recommend the following parameter selections. The positive parameter $\alpha$ should be chosen so that the true solution $u(x, y)$ satisfies the decay condition

$$
|u(x, y)| \leq C((x-a)(b-y))^{\alpha+1 / 2} .
$$

Then the parameter $h$ is given by $h=\left(\frac{\pi d}{\alpha N}\right)^{\frac{1}{2}}$, where the angle $d$ is taken to be $\frac{\pi}{2}$ for the problems considered.

The maximum absolute error between the numerical approximation $v_{N}(x, y)$ and the true solution $u(x, y)$ at the Sinc grid points is defined as $\|E\|_{g}$ and the maximum absolute error at the 100 equally spaced points is defined as $\|E\|_{u}$, where these equally spaced points $\left(x_{i}, y_{j}\right)$ are chosen to be $x_{i}=a_{1}+$ $\frac{i}{100}\left(b_{1}-a_{1}\right)$ and $y_{j}=a_{2}+\frac{j}{100}\left(b_{2}-a_{2}\right)$. The predicted asymptotic convergence rate is $O\left(e^{-(\pi d \alpha N)^{1 / 2}}\right)$. The code was run in double precision using the 
FORTRAN-77 compiler on a DECstation/UNIX computer. The numerical results for different $N$ are shown in the following tables.

Example 3.1.

$$
\frac{\partial^{2} u}{\partial x^{2}}+\frac{\partial^{2} u}{\partial y^{2}}=f(x, y), \quad u(x, y)=\left(1-x^{2}\right)^{\frac{7}{4}}(y-1)^{2}(4-y)^{2},
$$

where $\Omega=(-1,1) \times(1,4)$. The parameter $\alpha$ is chosen to be $\alpha=5 / 4$. The result is shown in Table 1 .

TABLE 1

\begin{tabular}{||c|c|c|c|c||}
\hline $\mathrm{N}$ & $h$ & $\|E\|_{g}$ & $\|E\|_{u}$ & asymptotic error \\
\hline 2 & 1.404963 & $1.0802 \mathrm{D}-1$ & $1.1046 \mathrm{D}-1$ & $2.9825 \mathrm{D}-2$ \\
\hline 4 & 0.993459 & $1.1282 \mathrm{D}-2$ & $1.1887 \mathrm{D}-2$ & $6.9620 \mathrm{D}-3$ \\
\hline 8 & 0.702481 & $1.0286 \mathrm{D}-3$ & $1.0048 \mathrm{D}-3$ & $8.8953 \mathrm{D}-4$ \\
\hline 16 & 0.496729 & $2.4315 \mathrm{D}-5$ & $2.3147 \mathrm{D}-5$ & $4.8469 \mathrm{D}-5$ \\
\hline 32 & 0.351241 & $9.5987 \mathrm{D}-8$ & $9.2376 \mathrm{D}-8$ & $7.9127 \mathrm{D}-7$ \\
\hline
\end{tabular}

\section{Example 3.2.}

$$
\frac{\partial^{2} u}{\partial x^{2}}+\frac{\partial^{2} u}{\partial y^{2}}=f(x, y), \quad u(x, y)=\left(x^{3 / 2}-x\right)\left(y^{3 / 2}-y\right),
$$

where $\Omega=(0,1) \times(0,1)$. This example is found in [2], where the authors used it to illustrate the ability of the Sinc method to solve singular problems, and where they compared it with the difficulties inherent in the standard finite difference or finite element methods presented in [12]. The parameter $\alpha$ is here chosen to be $\alpha=1 / 2$. The result is shown in Table 2 .

TABLE 2

\begin{tabular}{||c|c|c|c|c||}
\hline $\mathrm{N}$ & $h$ & $\|E\|_{g}$ & $\|E\|_{u}$ & asymptotic error \\
\hline 2 & 2.221441 & $2.5315 \mathrm{D}-4$ & $7.4670 \mathrm{D}-4$ & $1.0845 \mathrm{D}-1$ \\
\hline 4 & 1.570796 & $7.0055 \mathrm{D}-5$ & $1.2511 \mathrm{D}-4$ & $4.3214 \mathrm{D}-2$ \\
\hline 8 & 1.110721 & $7.7647 \mathrm{D}-6$ & $1.3159 \mathrm{D}-5$ & $1.1762 \mathrm{D}-2$ \\
\hline 16 & 0.785398 & $2.5963 \mathrm{D}-7$ & $5.1589 \mathrm{D}-7$ & $1.8674 \mathrm{D}-3$ \\
\hline 32 & 0.555360 & $1.7498 \mathrm{D}-9$ & $8.6613 \mathrm{D}-9$ & $1.3834 \mathrm{D}-4$ \\
\hline
\end{tabular}

Example 3.3.

$$
\frac{\partial^{2} u}{\partial x^{2}}+\frac{\partial^{2} u}{\partial y^{2}}=f(x, y), \quad u(x, y)=x y \ln x \ln y,
$$

where $\Omega=(0,1) \times(0,1)$. This example is also found in [2] and illustrates a logarithmic singularity. The parameter $\alpha$ can be chosen as large as possible in this case, i.e., $\alpha=1 / 2$. The result is shown in Table 3 . 
TABLE 3

\begin{tabular}{||c|c|c|c|c||}
\hline $\mathrm{N}$ & $h$ & $\|E\|_{g}$ & $\|E\|_{u}$ & asymptotic error \\
\hline 2 & 2.221441 & $3.9517 \mathrm{D}-3$ & $8.1485 \mathrm{D}-3$ & $1.0845 \mathrm{D}-1$ \\
\hline 4 & 1.570796 & $1.3048 \mathrm{D}-3$ & $2.0009 \mathrm{D}-3$ & $4.3214 \mathrm{D}-2$ \\
\hline 8 & 1.110721 & $1.8299 \mathrm{D}-4$ & $2.6689 \mathrm{D}-4$ & $1.1762 \mathrm{D}-2$ \\
\hline 16 & 0.785398 & $8.5641 \mathrm{D}-6$ & $1.7600 \mathrm{D}-5$ & $1.8674 \mathrm{D}-3$ \\
\hline 32 & 0.555360 & $7.9693 \mathrm{D}-8$ & $5.7605 \mathrm{D}-7$ & $1.3834 \mathrm{D}-4$ \\
\hline
\end{tabular}

\section{SOLUtion OF FOURTH-ORDER POISSON-LIKE PROBLEMS}

We now consider the fourth-order Poisson-like problem:

$$
\begin{aligned}
& \frac{\partial^{4} u}{\partial x^{4}}+\frac{\partial^{4} u}{\partial y^{4}}=f(x, y), \quad(x, y) \in \Omega \equiv\left(a_{1}, b_{1}\right) \times\left(a_{2}, b_{2}\right), \\
& \left.u\right|_{\partial \Omega}=\left.\frac{\partial u}{\partial x}\right|_{\partial \Omega}=\left.\frac{\partial u}{\partial y}\right|_{\partial \Omega}=0 .
\end{aligned}
$$

Smith et al. [6] have discussed the one-dimensional case of the above problem by using the Sinc-Galerkin method and got an accurate numerical solution to an order of $O\left(e^{-\sqrt{\pi d \alpha N}}\right)$.

Here we can still solve (4.1) by using the Sinc-collocation method with the orthogonalization technique described in $\S 3.2$.

Let $v_{N}(x, y)$ be defined by (3.8) with the choice $h_{1}=h_{2}=h$ and $N_{1}=$ $N_{2}=N$, where $v_{k_{1} k_{2}}$ will be determined by

$$
\sum_{k_{1}=-N}^{N} \frac{v_{k_{1} i_{2}}}{g_{k_{1}}} S_{k_{1}}^{\prime \prime \prime \prime}\left(x_{i_{1}}\right)+\sum_{k_{2}=-N}^{N} \frac{v_{i_{1} k_{2}}}{\bar{g}_{k_{2}}} \bar{S}_{k_{2}}^{\prime \prime \prime \prime}\left(y_{i_{2}}\right)=f_{i_{1} i_{2}} .
$$

Analogous to $\S 3.2$, a matrix representation of the equation (4.2) can be obtained in the form

$$
S_{x} D\left(\frac{1}{g(x)}\right) U+U D\left(\frac{1}{\bar{g}(y)}\right) S_{y}^{t}=W,
$$

where $S_{x}$ and $S_{y}$ are $m \times m$ matrices whose $(i k)$ th entries are $S_{k}^{\prime \prime \prime \prime}\left(x_{i}\right)$ and $\bar{S}_{k}^{\prime \prime \prime \prime}\left(y_{i}\right)$, respectively. The matrices $U$ and $W$ are also $m \times m$, with $(i k)$ th entries given by $v_{i k}$ and $f_{i k}$, respectively.

It is easy to verify from $(2.4)$ that

$$
S_{k}^{\prime \prime \prime \prime}\left(x_{i}\right)=g_{i}^{\prime \prime \prime \prime} \delta_{k i}^{(0)}+\frac{1}{h} a_{i}^{(1)} \delta_{k i}^{(1)}+\frac{1}{h^{2}} a_{i}^{(2)} \delta_{k i}^{(2)}+\frac{1}{h^{3}} a_{i}^{(3)} \delta_{k i}^{(3)}+\frac{1}{h^{4}} g_{i} \phi_{i}^{(4} \delta_{k i}^{(4)},
$$

where

$$
\begin{gathered}
a^{(1)}=4 g^{\prime \prime \prime} \phi^{\prime}+6 g^{\prime \prime} \phi^{\prime \prime}+4 g^{\prime} \phi^{\prime \prime \prime}+g \phi^{\prime \prime \prime \prime}, \\
a^{(2)}=6 g^{\prime \prime} \phi^{\prime^{2}}+12 g^{\prime} \phi^{\prime} \phi^{\prime \prime}+3 g \phi^{\prime{ }^{2}}+4 g \phi^{\prime} \phi^{\prime \prime \prime}, \\
a^{(3)}=4 g^{\prime} \phi^{\prime 3}+6 g \phi^{\prime^{2}} \phi^{\prime \prime},
\end{gathered}
$$

and the $\delta_{k i}^{(j)}, j=0, \ldots, 4$ are defined in (3.3)-(3.7).

Take

$$
g(x)=\left(\phi^{\prime}(x)\right)^{-\frac{3}{2}}
$$


then we get $a^{(3)}(x)=0$. Direct verification, using (3.17), gives $a^{(1)}(x)=0$ and $a^{(2)}(x)=-\frac{5}{2} \phi^{\prime \frac{5}{2}}(x)$. It should be noted that, by using (4.4) and (3.17), we can prove that

It then follows that

$$
g^{\prime \prime \prime \prime}=\frac{9}{16} \phi^{\prime \frac{5}{2}}
$$

$$
S_{k}^{\prime \prime \prime \prime}\left(x_{i}\right)=\frac{1}{h^{4}} \phi_{i}^{\prime \frac{5}{2}}\left\{\delta_{k i}^{(4)}-\frac{5}{2} h^{2} \delta_{k i}^{(2)}+\frac{9}{16} h^{4} \delta_{k i}^{(0)}\right\} .
$$

Similarly, we get

$$
\bar{S}_{k}^{\prime \prime \prime \prime}\left(y_{i}\right)=\frac{1}{h^{4}} \bar{\phi}_{i}^{\prime \frac{5}{2}}\left\{\delta_{k i}^{(4)}-\frac{5}{2} h^{2} \delta_{k i}^{(2)}+\frac{9}{16} h^{4} \delta_{k i}^{(0)}\right\}
$$

by taking

$$
\bar{g}(y)=\left(\bar{\phi}^{\prime}(y)\right)^{-\frac{3}{2}} .
$$

Using the same procedure as in $\S 3.2$, we easily obtain

$$
A_{x} Y+Y A_{y}=h^{4} W^{\prime} \text {. }
$$

But now

$$
\begin{gathered}
A_{x}=D\left(\phi^{\prime 2}(x)\right) A D\left(\phi^{\prime 2}(x)\right), \quad A_{y}=D\left(\bar{\phi}^{\prime 2}(y)\right) A D\left(\bar{\phi}^{\prime 2}(y)\right), \\
Y=D\left(\phi^{\prime-\frac{1}{2}}(x)\right) U D\left(\bar{\phi}^{\prime-\frac{1}{2}}(y)\right), \\
W^{\prime}=D\left(\phi^{\prime-\frac{1}{2}}(x)\right) W D\left(\bar{\phi}^{\prime-\frac{1}{2}}(y)\right),
\end{gathered}
$$

and

(4.11) $A=\left(a_{i k}\right)=\left(\delta_{k i}^{(4)}-\frac{5}{2} h^{2} \delta_{k i}^{(2)}+\frac{9}{16} h^{4} \delta_{k i}^{(0)}\right)=I^{(4)}-\frac{5}{2} h^{2} I^{(2)}+\frac{9}{16} h^{4} I^{(0)}$.

Since the matrix $I^{(4)}$ is positive definite [6], we can show, in the same way as before, that both of the matrices $A_{x}$ and $A_{y}$ are symmetric positive definite, and furthermore, under the assumption that $h_{1}=h_{2}=h, N_{1}=N_{2}=N$, that we have $A_{y}=\kappa^{4} A_{x}$. Hence, the same procedure of our orthogonalization technique described in $\S 3.2$ can be applied to the Poisson-like problem (4.1), except that the matrix $Y^{\prime}$ in the equation (3.23) must be replaced by

$$
Y^{\prime}=\left(y_{i k}^{\prime}\right)=\left(\frac{h^{4} z_{i k}}{\lambda_{i}+\kappa^{4} \lambda_{k}}\right) \text {. }
$$

Also, the positive parameter $\alpha$ should be selected to satisfy

$$
|u(x, y)| \leq C((x-a)(b-y))^{\alpha+\frac{3}{2}} .
$$

Here we give some numerical results, where $\|E\|_{g},\|E\|_{u}$, and $d$ are defined as before.

\section{Example 4.1.}

$$
\frac{\partial^{4} u}{\partial x^{4}}+\frac{\partial^{4} u}{\partial y^{4}}=f(x, y), \quad u(x, y)=\left(1-x^{2}\right)^{3}(y-1)^{3}(4-y)^{3}
$$


where $\Omega=(-1,1) \times(1,4)$. This is a problem whose solution is known analytically. The parameter $\alpha$ is chosen to be $\alpha=3 / 2$. The result is shown in Table 4.

TABLE 4

\begin{tabular}{||c|c|c|c|c||}
\hline $\mathrm{N}$ & $h$ & $\|E\|_{g}$ & $\|E\|_{u}$ & asymptotic error \\
\hline 2 & 1.282550 & $9.4962 \mathrm{D}-1$ & $9.5118 \mathrm{D}-1$ & $2.1330 \mathrm{D}-2$ \\
\hline 4 & 0.906900 & $2.7328 \mathrm{D}-2$ & $5.9729 \mathrm{D}-2$ & $4.3334 \mathrm{D}-3$ \\
\hline 8 & 0.641275 & $3.0343 \mathrm{D}-3$ & $3.7935 \mathrm{D}-3$ & $4.5496 \mathrm{D}-4$ \\
\hline 16 & 0.453450 & $7.3851 \mathrm{D}-5$ & $7.3851 \mathrm{D}-5$ & $1.8779 \mathrm{D}-5$ \\
\hline 32 & 0.320637 & $9.3745 \mathrm{D}-6$ & $9.4706 \mathrm{D}-6$ & $2.0699 \mathrm{D}-7$ \\
\hline
\end{tabular}

Example 4.2.

$$
\frac{\partial^{4} u}{\partial x^{4}}+\frac{\partial^{4} u}{\partial y^{4}}=f(x, y), \quad u(x, y)=y^{3}(\sin x)^{3} \ln ^{3} y
$$

where $\Omega=(0, \pi) \times(0,1)$. This problem is singular in $y$. The parameter $\alpha$ is selected to be $\alpha=3 / 2$. The result is shown in Table 5 .

TABLE 5

\begin{tabular}{||c|c|c|c|c||}
\hline $\mathrm{N}$ & $h$ & $\|E\|_{g}$ & $\|E\|_{u}$ & asymptotic error \\
\hline 2 & 1.282550 & $5.8964 \mathrm{D}-3$ & $8.3871 \mathrm{D}-3$ & $2.1330 \mathrm{D}-2$ \\
\hline 4 & 0.906900 & $2.8201 \mathrm{D}-3$ & $2.9605 \mathrm{D}-3$ & $4.3334 \mathrm{D}-3$ \\
\hline 8 & 0.641275 & $5.4495 \mathrm{D}-4$ & $5.4531 \mathrm{D}-4$ & $4.5496 \mathrm{D}-4$ \\
\hline 16 & 0.453450 & $2.9301 \mathrm{D}-5$ & $2.9605 \mathrm{D}-5$ & $1.8779 \mathrm{D}-5$ \\
\hline 32 & 0.320637 & $2.5942 \mathrm{D}-7$ & $2.6026 \mathrm{D}-7$ & $2.0699 \mathrm{D}-7$ \\
\hline
\end{tabular}

\section{Example 4.3.}

$$
\frac{\partial^{4} u}{\partial x^{4}}+\frac{\partial^{4} u}{\partial y^{4}}=f(x, y), \quad u(x, y)=(x(1-x))^{\frac{7}{2}}(y(2-y))^{\frac{10}{3}},
$$

where $\Omega=(0,1) \times(0,2)$. This example illustrates the highest degree of singularity ( $u$ has fourth partial derivatives which are undefined on the boundary.) The parameter $\alpha$ is chosen to be $\alpha=11 / 6$. The result is shown in Table 6 .

TABLE 6

\begin{tabular}{||c|c|c|c|c||}
\hline $\mathrm{N}$ & $h$ & $\|E\|_{g}$ & $\|E\|_{u}$ & asymptotic error \\
\hline 2 & 1.160110 & $8.5673 \mathrm{D}-4$ & $8.5673 \mathrm{D}-4$ & $1.4211 \mathrm{D}-2$ \\
\hline 4 & 0.820322 & $1.3176 \mathrm{D}-5$ & $3.4646 \mathrm{D}-5$ & $2.4402 \mathrm{D}-3$ \\
\hline 8 & 0.580055 & $1.0888 \mathrm{D}-6$ & $1.4810 \mathrm{D}-6$ & $2.0196 \mathrm{D}-4$ \\
\hline 16 & 0.410161 & $1.5501 \mathrm{D}-8$ & $1.6013 \mathrm{D}-8$ & $5.9544 \mathrm{D}-6$ \\
\hline 32 & 0.290027 & $3.4867 \mathrm{D}-10$ & $3.4871 \mathrm{D}-10$ & $4.0786 \mathrm{D}-8$ \\
\hline
\end{tabular}




\section{ACKNOWLEDGMENTS}

The author thankfully acknowledges the supportive encouragement of Professor Frank Stenger during the preparation of this manuscript and appreciates the constructive remarks of the referee.

\section{BIBLIOGRAPHY}

1. E. P. Doolan, J. J. H. Miller and W. H. A. Schilders, Uniform numerical methods for problems with initial and boundary layers, Boole Press, Dublin, 1980.

2. K. L. Bowers and J. Lund, Numerical solution of singular Poisson problems via the SincGalerkin method, SIAM J. Numer. Anal. 24 (1987), 36-51.

3. J. Lund, Symmetrization of the Sinc-Galerkin method for boundary value problems, Math. Comp. 47 (1986), 571-588.

4. J. Lund, K. L. Bowers, and K. M. McArthur, Symmetrization of the Sinc-Galerkin method with block techniques for elliptic equations, IMA J. Numer. Anal. 9 (1989), 29-46.

5. L. Lundin and F. Stenger, Cardinal-type approximations of a function and its derivatives, SIAM J. Math. Anal. 10 (1979), 139-160.

6. R. C. Smith, K. L. Bowers, and J. Lund, Efficient numerical solution of fourth-order problems in the modeling of flexible structures, Computation and Control ( $\mathrm{K}$. Bowers and J. Lund, eds.), Birkhäuser, Boston, 1988, pp. 283-297.

7. F. Stenger, Integration formulas via the trapezoidal formula, J. Inst. Math. Appl. 12 (1973), 103-114.

8. __ $A$ "Sinc-Galerkin" methoa of solution of boundary value problems, Math. Comp. 33 (1979), 85-109.

9. _ Numerical methods based on Whittaker cardinal, or Sinc functions, SIAM Rev. 23 (1981), 165-224.

10. __ Sinc methods of approximate solution of partial differential equations, Advances in Computer Methods for Partial Differential Equations, Philadelphia, 1984, pp. 244-251.

11. __ Numerical methods based on Sinc and analytic functions, Springer-Verlag, New York, 1993.

12. A. Weiser, S. C. Eisenstat, and M. H. Schultz, On solving elliptic problems to moderate accuracy, SIAM J. Numer. Anal. 17 (1980), 908-929.

Department of Computer Science, University of Utah, Salt Lake City, Utah 84112

E-mail address: gyin@cs.utah.edu 